\title{
MELHORIAS NA DISCIPLINA DE CUSTOS IDENTIFICADAS POR MEIO DA UTILIZAÇÃO CONJUNTA DO MODELO KANO DE QUALIDADE E DA MATRIZ DE IMPORTÂNCIA E DESEMPENHO
}

\section{IMPROVEMENT IN THE SUBJECT OF COSTS USING THE KANO MODEL OF QUALITY AND THE MATRIX OF IMPORTANCE AND PERFORMANCE}

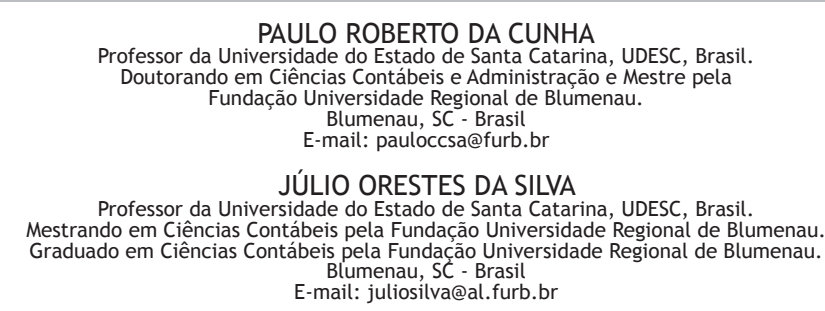

PAULO ROBERTO DA CUNHA

E-mail: pauloccsa@furb.b

E-mail: juliosilva@al.furb.br

\author{
ROBERTO CARLOS KLANN \\ Professor da Fundação Universidade Regional de Blumenau, FURB, Brasil. \\ Doutorando em Ciências Contábeis e Administração e Mestre pela \\ Fundação Universidade Regional de Blumenau. \\ Blumenau, SC-Brasil
E-mail: rklann@al furb.br \\ JORGE EDUARDO SCARPIN
Professor da Fundação Universidade Regional de Blumenau, FURB, Brasil. \\ Doutor em Controladoria e Contabilidade pela Universidade de São Paulo. \\ Blumenau, SC - Brasil
}

\section{RESUMO}

O objetivo desta pesquisa foi aplicar a técnica do modelo Kano de Qualidade Atrativa e Obrigatória em conjunto com a Matriz de Importância e Desempenho para identificar oportunidades de melhoria na disciplina de custos de diferentes cursos de graduação. 0 delineamento metodológico caracterizase por um estudo descritivo, por um levantamento com uma abordagem quantitativa e por uma etapa prévia qualitativa. A população da pesquisa foi definida de forma intencional, em linha com o objeto de estudo, em que os sujeitos foram os alunos que cursam a disciplina de custos nos cursos de administração, ciências contábeis, ciências econômicas, engenharia de produção e moda de uma universidade do Sul do país, perfazendo o total de 289 alunos. A amostra por acessibilidade foi de 214 alunos, ou seja, 74\%. O instrumento utilizado para coleta de dados foi um questionário com base no modelo Kano de qualidade atrativa e obrigatória adaptado de Walter, Tontini e Domingues (2005), complementado por atributos identificados por meio da aplicação da técnica de focus group. 0 instrumento contemplou a possibilidade de análise de 23 atributos, agrupados em cinco categorias: capacitação dos professores, métodos de ensino, atitude, conteúdo e infra-estrutura. Os resultados evidenciaram que os atributos prioritários para melhoria na disciplina de custos nos diferentes cursos analisados são a modernidade dos laboratórios de informática e a infraestrutura da sala de aula.

Palavras-chave: Custos. Ensino superior. Atributos de qualidade. Modelo Kano de qualidade atrativa e obrigatória. Matriz de importância e desempenho.

\section{ABSTRACT}

The purpose of this study was apply the technique of the Kano Model of Quality and Attractive Required in conjunction with the Matrix of Significance and Performance to identify opportunities for improvement in the subject of costs in different undergraduate courses. The methodological design is characterized by a descriptive study and survey research with a quantitative approach and a prior qualitative step. The research population was intentionally set in line with the object of study: the subjects were the students who study the subject of costs in the courses of administration, accounting, economics, engineering, production and fashion from a university of the South of the country, summing up 289 students. The sample by accessibility was 214 students (74\%). The instrument used for data collection was a questionnaire based on Kano model of attractive and binding quality, adapted from Walter, Tontini and Domingues (2005), complemented by attributes identified by applying the technique of focus group. The instrument contemplated the possibility of analyzing 23 attributes, grouped into five categories: teacher training, teaching methods, attitude, content and infrastructure. The results showed that priority attributes in the subject of costs in different courses are modernity of the computer labs and infrastructure of the classroom.

Keywords: Costs. Higher Education. Quality attributes. Kano model of attractive and binding quality. Matrix of importance and performance. 


\section{INTRODUÇÃO}

As Instituições de Ensino Superior (IES) configuram-se atualmente num setor da economia altamente competitivo, em que a oferta de cursos em diversas regiões do país se revela superior à demanda. Conforme Walter, Tontini e Domingues (2005), essa realidade é reflexo da facilidade de abertura de IES proporcionada pela Lei de Diretrizes e Bases (LDB) de 1996. Assim, apesar da regulação governamental, o mercado educacional aproxima-se cada vez mais de um setor no qual a qualidade dos serviços e a satisfação dos clientes são fundamentais para a sobrevivência das IES.

Nesse sentido, o valor agregado aos serviços tornou-se um dos diferenciais competitivos de mercado em decorrência desse aumento das IES. Assegurar a qualidade no ensino superior configura-se como uma das principais estratégias de sobrevivência destas IES (SILVEIRA; APPIO; DOMINGUES, 2008).

Para prestar um serviço de qualidade, é prudente identificar quais são os atributos considerados importantes na prestação do serviço pelos clientes, que, no caso de uma IES, configuram-se nos alunos. Essa preocupação com a qualidade do serviço pode se revelar um instrumento gerencial significativo às IES.

Alunos satisfeitos com os serviços internos da IES e com os cursos que esta oferece influenciarão positivamente a percepção que a sociedade e futuros alunos têm a seu respeito, aumentando a demanda. Já uma percepção negativa terá efeito contrário. Assim, identificar como os diferentes atributos da IES e seus cursos afetam a satisfação dos alunos e qual seu nível atual de satisfação torna-se crítico para o gerenciamento eficaz da IES (WALTER; TONTINI; DOMINGUES, 2005).

Estudos que envolvem a avaliação da qualidade ligada ao ensino superior tem sido objeto de diversas pesquisas, como Figueredo (2005); Marzo-Navarro, Morales e Moncloa (2005); Pedraja-Iglesias e Rivera-Torres (2005); Walter, Tontini e Domingues (2005); Zani (2005); Fiebig (2006); Mondini (2006); Walter (2006); Deschamps (2007); Mainardes (2007); Mondini, Silveira e Tontini (2007); Zwierewicz (2008).

No estudo de Walter, Tontini e Domingues (2005), o objetivo foi mostrar resultados parciais de uma pesquisa em andamento que utiliza o Modelo Kano de Qualidade Atrativa e Obrigatória e outros métodos de análise na identificação de oportunidades de melhoria em Instituições de Ensino Superior (IES). Neste estudo foram analisados 20 atributos do curso de administração da Universidade Regional de Blumenau/SC, analisados por meio de uma pesquisa quantitativa, com uma amostra de 362 alunos das diferentes habilitações, turnos e fases. Para a identificação de oportunidades de melhoria, foram utilizados o Modelo Kano e a Matriz de Importância $x$ Desempenho. Com o uso conjunto dos dois métodos foi possível uma melhor identificação dos atributos prioritários para melhoria, que foram a quantidade de vagas no estacionamento, a agilidade do laboratório de impressão, a modernidade dos laboratórios de informática e a relação entre teoria e prática nas disciplinas. Os atributos considerados críticos pela Matriz de Importância x Desempenho tiveram 
sua prioridade reduzida por serem considerados atrativos pelo Modelo Kano. Os resultados demonstraram a potencialidade de aplicação conjunta dos dois métodos na identificação de oportunidades de melhoria nas IES.

Outro estudo que buscou identificar melhorias em um curso de ensino superior por meio da utilização conjunta do modelo Kano de qualidade e da matriz de importância e desempenho foi Parisotto et al (2008). O objetivo deste estudo foi identificar oportunidades de melhorias no curso de Ciências Contábeis de uma IES, com a utilização do modelo Kano de qualidade em conjunto com a matriz de importância $x$ desempenho. Utilizou-se a aplicação de questionário sobre atributos previamente definidos a alunos do curso de ciências contábeis, matriculados entre a $4^{\mathrm{a}}$ e $10^{\mathrm{a}}$ fase. Dos 251 alunos matriculados, 120 responderam ao questionário. Com a pesquisa, foi possível concluir que na amostra investigada, os atributos prioritários para melhoria no curso de ciências contábeis desta IES são a colocação no mercado para alunos do curso, clareza na exposição dos assuntos das disciplinas, utilização adequada dos métodos de ensino, qualidade do curso em relação ao preço atual da mensalidade, relevância das disciplinas para a vida profissional, atualização dos conteúdos das disciplinas e comprometimento dos professores com o aprendizado dos alunos.

Porém, Silveira, Appio e Domingues (2008) destacam que os estudos dos atributos de qualidade no ensino superior podem ser identificados não só em relação aos cursos que a IES oferece no nível de graduação e de pós-graduação mas, também, no que tange às disciplinas destes cursos. Nesse sentido, não foram identificados estudos sobre a qualidade no ensino da disciplina de custos, bem como estudos sobre a qualidade no ensino de uma disciplina em diferentes cursos, sendo que, para tal, elegeu-se a disciplina de custos. Nessa busca de identificação de atributos de qualidade, uma técnica utilizada é ao modelo Kano de qualidade atrativa e obrigatória aplicado em conjunto com a matriz de importância e desempenho.

Garver (2003, p. 456) destaca que “durante décadas, a análise por meio da matriz de importância $x$ desempenho tem sido discutida como uma ferramenta para avaliar a posição competitiva de uma empresa no mercado, para identificar oportunidades de melhoria e para orientar os esforços de planejamento estratégico". Contudo, Matzler et al. (2004) destacam que devido ao relacionamento assimétrico e não-linear entre o desempenho dos diferentes atributos de um produto e a satisfação do consumidor, a validade de sua aplicação tem sido questionada. Nesse sentido, Tontini e Silveira (2005) destacam que o modelo Kano de Qualidade Atrativa e Obrigatória apresentase como uma alternativa para identificar o efeito do desempenho dos atributos na satisfação dos clientes por considerar a não-linearidade entre o desempenho e satisfação.

Diante do exposto, levanta-se a seguinte questão de pesquisa para o presente estudo: Quais são os atributos de qualidade e as oportunidades de melhoria revelados pelos discentes de uma IES para a disciplina de custos em diferentes cursos de graduação?

Assim, o objetivo desta pesquisa é aplicar a técnica do modelo Kano de Qualidade Atrativa e Obrigatória em conjunto com a Matriz de Importância e Desempenho para 
identificar oportunidades de melhoria na disciplina de custos de diferentes cursos de graduação.

Apesar de o modelo Kano de Qualidade Atrativa e Obrigatória ter sido desenvolvido para se ter uma visão sobre quais atributos do produto ou serviço de uma organização devem ser melhorados para proporcionar satisfação aos clientes, sua aplicação na área de ensino em conjunto com a Matriz de Importância e Desempenho já foi contemplada em estudos como o de Walter, Tontini e Domingues (2005) e Parisotto et al (2008). Justifica-se a utilização desses modelos para identificar possibilidades de melhorias numa disciplina, ao considerar que os achados podem contribuir para ações que se façam necessárias, tanto pelo professor da disciplina quanto pela coordenação do curso.

O artigo está estruturado em quatro tópicos, iniciando com esta introdução. Na sequência faz-se uma incursão teórica acerca dos métodos de identificação de oportunidades de melhoria, especificamente da Matriz de Importância e Desempenho e do modelo Kano de Qualidade Atrativa e Obrigatória. Em seguida, descreve-se o método e procedimento da pesquisa e a descrição e análise dos dados. Por fim, apresentam-se as conclusões da pesquisa realizada.

\section{A MATRIZ DE IMPORTÂNCIA E DESEMPENHO}

A análise de importância versus desempenho é uma técnica de identificação de oportunidades de melhoria, introduzida originalmente por Martilla e James (1977). Essa técnica de análise permite à organização ter uma visão sobre quais atributos de seu produto ou serviço devem ser melhorados para proporcionar satisfação aos clientes (WALTER, 2006).

Um gráfico bi-dimensional relaciona o desempenho atual de um atributo com seu grau de importância. Por meio de um questionário tipo Likert, a matriz dá uma clara visualização, a partir do posicionamento dos atributos nos quadrantes do gráfico, de quais atributos devem ser melhorados, ignorados, mantidos ou estão com desempenho acima do esperado (FIGUEREDO, 2005).

Matzler et al. (2004, p. 272) expõem que "os dados de pesquisas sobre satisfação são utilizados para construir uma matriz bi-dimensional, onde é retratada a importância ao longo do eixo-x e desempenho (satisfação) ao longo do y", como demonstrado na Figura 1. Os consumidores avaliam qual a importância para eles e o desempenho da organização em relação ao atendimento de suas expectativas para cada atributo.

A importância é atribuída geralmente por meio de uma escala direta (ex: 1 a 5) ou análise de regressão, em que a importância é dada pelo coeficiente de uma regressão linear entre o desempenho do atributo e a satisfação geral com o serviço. 0 desempenho é atribuído por meio de escala direta (ex: 1 a 10) ou de maneira relativa à concorrência ou ao desempenho esperado (ex: Muito Pior, Pior, Igual, Melhor, Muito 
Melhor) (GARVER, 2003).

Matriz de importância $\mathbf{x}$ desempenho

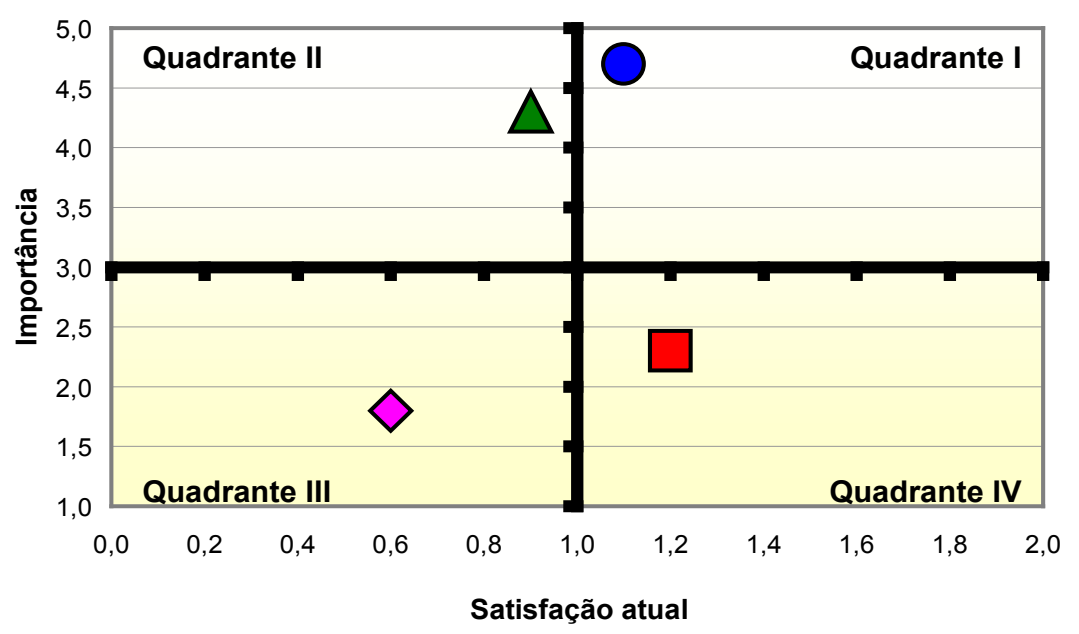

Figura 1 - Matriz de Importância e Desempenho Fonte: Adaptado de Garver (2003).

A Figura 1 apresenta a Matriz de Importância e Desempenho dividida em quatro quadrantes, a qual é explicada por Tontini et al. (2004). Um atributo que se situar no Quadrante I terá alta importância com alto desempenho, representando uma possível vantagem competitiva. Nesse caso, a organização deve manter o bom trabalho. Um tributo situado no Quadrante II possui alta importância, mas baixo desempenho. Neste contexto, o atributo deve receber atenção imediata da organização para melhorar a satisfação geral. O Quadrante III contém os atributos com baixa importância e baixo desempenho, não sendo necessário concentrar esforço adicional nesses atributos. O Quadrante IV concentra os atributos com alto desempenho, porém, com baixa importância. Nesse caso, a empresa pode estar desperdiçando recursos que poderiam ser mais bem aproveitados em outro item ou no desempenho de outro atributo.

\section{MODELO KANO DE QUALIDADE ATRATIVA E OBRIGATÓRIA}

O Modelo Kano de Qualidade Atrativa e Obrigatória (KANO et al., 1984; BERGER et al., 1993, MATZLER et al., 1996) faz distinção entre três tipos de atributos de produtos ou serviços que influenciam a satisfação do cliente: obrigatórios, unidimensionais e atrativos.

Os atributos obrigatórios preenchem as funções básicas de um produto. Se estes atributos não estiverem presentes ou seu desempenho é insuficiente, os clientes ficarão extremamente insatisfeitos. Por outro lado, se estes atributos estiverem 
presentes ou são suficientes, eles não trazem satisfação. Os clientes veem estes atributos como pré-requisitos (KANO et al., 1984).

Tontini (2003a) utiliza como exemplo a limpeza em um restaurante que é vista como necessária pelos clientes. Se ela for insuficiente, trará insatisfação, porém, se presente, não trará satisfação. Os atributos obrigatórios geralmente não são exigidos explicitamente pelos clientes, pois eles os consideram como inerentes (TONTINI, 2003a).

Outro exemplo exposto por Walter, Tontini e Domingues (2005) é a ética do professor em sala de aula em relação à universidade. Caso ele fale bem da instituição, não trará um grande diferencial motivacional para os alunos. Isto irá contribuir apenas para um bom clima de aprendizado. Já, se o professor criticar esta universidade trará um clima de insatisfação geral aos alunos e, por consequência, uma imagem negativa do professor e da universidade.

Quanto aos atributos unidimensionais, a satisfação do cliente é proporcional ao nível de desempenho: quanto maior o nível de desempenho, maior será a satisfação do cliente e vice-versa (KANO et al., 1984). Como exemplo, Tontini (2003a) expõe que para uma determinada classe de automóvel, se o gasto de combustível por quilômetro rodado está abaixo de um certo nível, quanto menor for o consumo, tanto maior será a satisfação do cliente. Se o gasto com combustível por quilômetro rodado está acima deste nível, o cliente ficará insatisfeito. Quanto menor for a quilometragem por litro de combustível, tanto maior será a insatisfação do cliente referente a este requisito. Neste exemplo, o nível zero é a média da indústria para aquela classe de automóvel. Geralmente atributos unidimensionais são exigidos explicitamente pelos clientes.

Walter, Tontini e Domingues (2005) destacam outro exemplo em relação à prestação de serviços em uma secretaria universitária. Quanto melhor for o atendimento nos aspectos de gentileza e cortesia na secretaria, maior será a satisfação do aluno. Quanto menos gentileza e cortesia houver no atendimento, maior será a insatisfação.

Em relação aos atributos atrativos, Kano et al. (1984) expõem estes atributos são pontos-chave para a satisfação do cliente. $O$ atendimento destes atributos traz uma satisfação mais que proporcional. Porém, eles não trazem insatisfação se não forem atendidos. Tontini (2003a) exemplifica da seguinte forma: para um automóvel popular, a presença de ar condicionado como equipamento de série traz satisfação. Já sua ausência não traz insatisfação. Atributos atrativos não são nem expressos explicitamente e nem esperados pelo cliente.

Outro exemplo exposto por Walter, Tontini e Domingues (2005) é se a universidade proporcionar um curso extra de extensão para suprir uma necessidade detectada em uma determinada turma, isso certamente trará satisfação. Se o curso extra não for oferecido, isso não trará insatisfação aos alunos. Os atributos obrigatórios, unidimensionais e atrativos são sintetizados na Figura 2. 


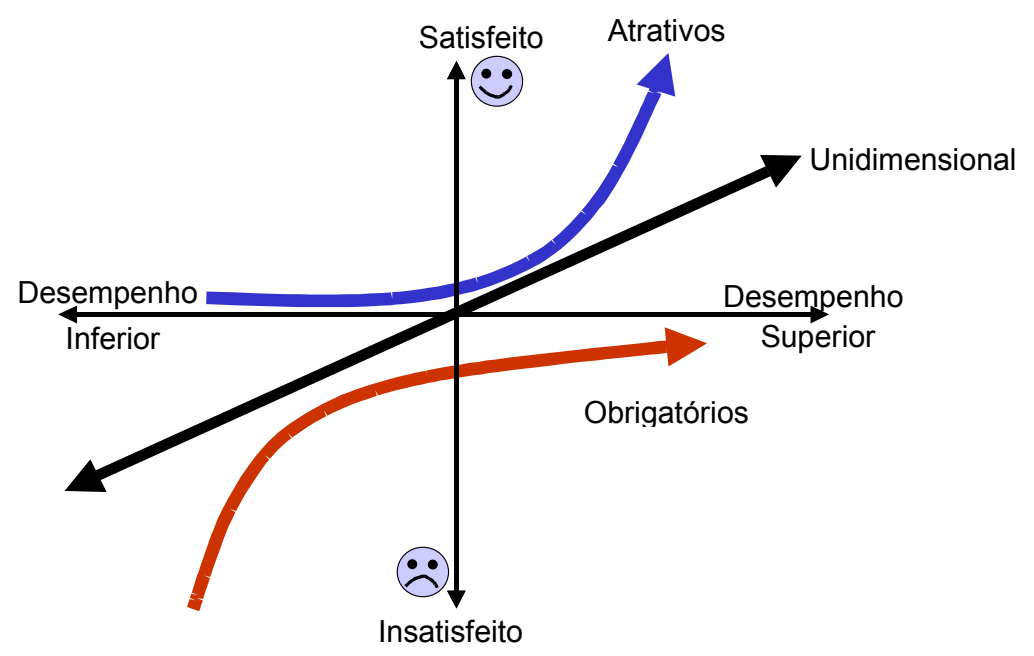

Figura 2 - Modelo Kano de qualidade atrativa e obrigatória

Fonte: Adaptado de Matzler et al. (1996).

Além desses três atributos mencionados por Kano et al. (1984), outros dois atributos são identificados por Tontini (2003a): neutros e reversos. Tontini (2003a) descreve que atributos neutros são aqueles cuja presença não traz satisfação e a sua ausência não traz insatisfação. São aqueles atributos que nunca, ou apenas raramente, são usados pelo cliente ou que o cliente não sabe como utilizá-lo.

Os atributos reversos são aqueles cuja presença traz insatisfação. Por exemplo, para algumas pessoas a presença de TVs em restaurantes não são bem-vindas. Para estes clientes a TV pode ser considerada um atributo reverso (TONTINI, 2003a).

Segundo o Modelo Kano, alguns atributos podem trazer mais satisfação que outros quando estão presentes. Por sua vez, alguns atributos obrigatórios podem trazer mais insatisfação do que outros quando tiverem desempenho insuficiente ou não estiverem presentes. A identificação do grau de satisfação ou insatisfação que o desempenho de um atributo específico pode causar ao cliente é de suma importância para garantir um alto grau de competitividade no mercado. Entretanto, o modelo original de Kano não identifica o grau de satisfação dos clientes (WALTER, 2006).

Assim, Berger et al. (1993) desenvolveram uma forma de identificar o grau de satisfação ou insatisfação que a presença/suficiência, ou ausência/insuficiência de um atributo causa aos clientes, denominado Coeficiente de Satisfação do Cliente (CSC). Walter, Tontini e Domingues (2005) descrevem que o CSC indica se a satisfação do cliente pode ser aumentada ao se melhorar um requisito do produto ou serviço, ou se apenas evitar-se-á que o cliente fique insatisfeito. O CSC identifica a proporção de clientes que afirmaram ficar satisfeitos com a presença ou suficiência de um atributo, e a proporção de clientes que afirmaram ficar insatisfeitos com sua ausência ou insuficiência. Porém, ele não considera o grau de satisfação ou insatisfação que um atributo pode trazer. 
Tontini (2003b) desenvolveu uma metodologia modificada para identificação dos atributos dentro do Modelo Kano de Qualidade Atrativa e Obrigatória, possibilitando a determinação do grau de satisfação ou insatisfação que a existência ou inexistência de um atributo pode trazer aos consumidores, conforme demonstrada na Figura 3.

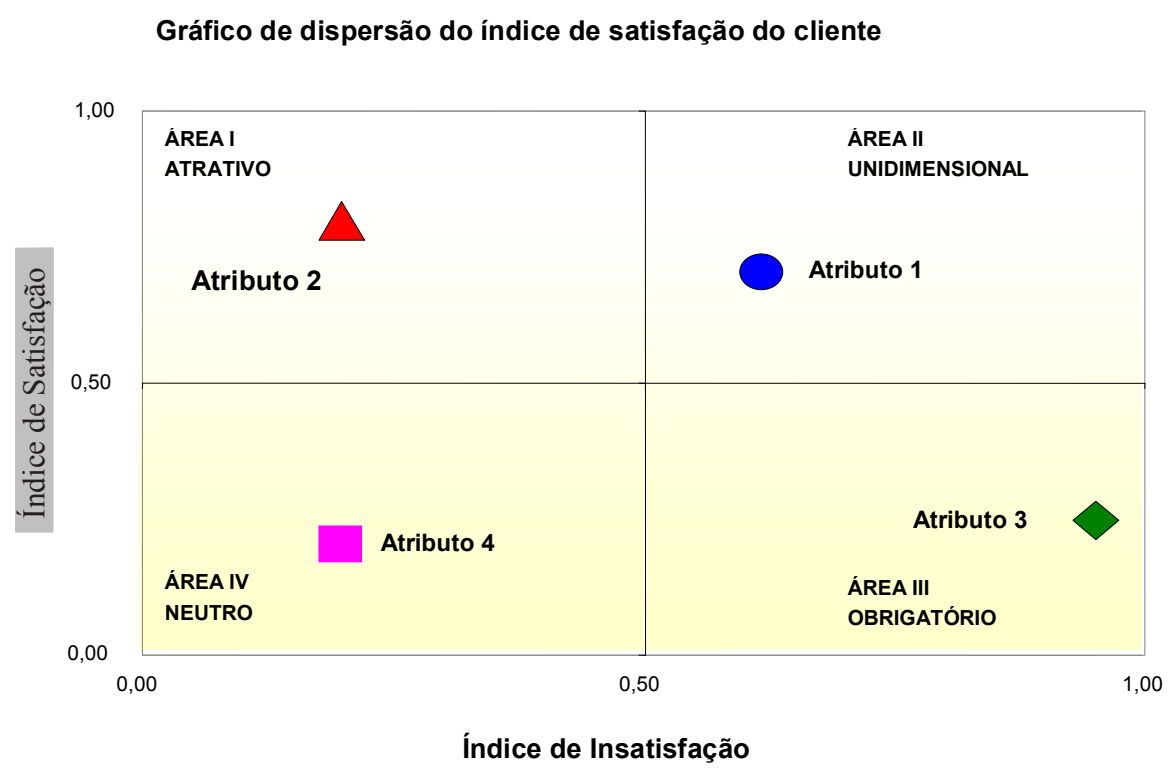

Figura 3 - Gráfico de classificação dos atributos segundo o modelo Kano de Qualidade Atrativa e Obrigatória

Fonte: Adaptado de Tontini (2003b).

Nessa metodologia, Walter, Tontini e Domingues (2005) explicam que o respondente avalia seu sentimento em uma escala variando de muito insatisfeito $(-3)$ a muito satisfeito $(+3)$, gerando um gráfico que reflete a média de satisfação ou insatisfação com a variação de desempenho dos atributos. Além da modificação no questionário, Tontini (2003b) introduziu uma maneira modificada de calcular o coeficiente de satisfação do cliente de Berger et al. (1993). Dois índices, variando entre 0 e 1 em uma escala padronizada, são calculados para cada atributo: IS - Índice de Satisfação, que é a média da satisfação com a questão positiva; II - Índice de Insatisfação, que é a média da satisfação (ou insatisfação) com a pergunta negativa. Para classificação dos atributos, os índices são apresentados em um gráfico de dispersão dividido em quatro áreas, conforme demonstrado na Figura 3.

Na área I, estão os atributos atrativos, pois eles trazem maior satisfação (quando têm desempenho superior) que insatisfação (quando têm desempenho inferior). Na área II, estão os atributos considerados unidimensionais, pois eles trazem tanto satisfação quando apresentam desempenho superior como insatisfação quando apresentam desempenho inferior.

$\mathrm{Na}$ área III, estão os atributos obrigatórios, que trazem alta insatisfação quando insuficientes, porém, não trazem grande satisfação quando suficientes. Na área IV, estão os atributos neutros (WALTER; TONTINI; DOMINGUES, 2005). 


\section{MÉTODO E TÉCNICAS DE PESQUISA}

A presente pesquisa caracteriza-se quanto aos objetivos como descritiva. Gil (2002) expõe que a pesquisa descritiva tem como objetivo descrever as características de determinada população ou fenômeno ou estabelecer relações entre variáveis. Assim, esta pesquisa busca descrever as oportunidades de melhorias na disciplina de custos ministrada em diferentes cursos com a utilização do modelo Kano de Qualidade Atrativa e Obrigatória em conjunto com a Matriz de Importância e Desempenho.

Pelos procedimentos empregados, caracteriza-se como uma pesquisa de levantamento, visto que se busca nos alunos matriculados na disciplina de custos, ofertadas em diversos cursos de uma universidade localizada na região Sul do Brasil, identificar as oportunidades de melhorias da disciplina.

Quanto à abordagem, a pesquisa caracteriza-se como qualitativa e quantitativa. Richardson (1989, p. 80) destaca que "os estudos que empregam uma metodologia qualitativa podem descrever a complexidade de determinado problema, analisar a interação de certas variáveis, compreender e classificar processos dinâmicos vividos por grupos sociais."

Boudon (1989, p. 24) afirma que "as pesquisas quantitativas podem ser definidas como as que permitem recolher, num conjunto de elementos, informações comparáveis entre um elemento e outro."

A população de pesquisa foi definida de forma intencional, por conveniência e a amostra foi por acessibilidade. A seleção da universidade se justifica uma vez que a mesma encontra-se entre as maiores e mais conceituadas universidades da região Sul, em que disciplinas de custos são ministradas em cinco cursos, contando com nove turmas no momento da realização da pesquisa, conforme exposto no Quadro 1.

\begin{tabular}{|c|c|c|c|}
\hline Turma & Nome da disciplina & Curso & Semestre \\
\hline 1 & Custo Aplicado à Administração & Administração & 3 \\
\hline 2 & Custo Aplicado à Administração & Administração & 3 \\
\hline 3 & Custo Aplicado à Administração & Administração & 3 \\
\hline 4 & Análise de Custos & Ciências Contábeis & 4 \\
\hline 5 & Contabilidade de Custos & Ciências Contábeis & 3 \\
\hline 6 & Análise de Custos & Ciências Econômicas & 7 \\
\hline 7 & Custos Industriais & Engenharia de Produção & 8 \\
\hline 8 & Gestão Estratégica de Custos & Engenharia de Produção & 9 \\
\hline 9 & \multicolumn{2}{|c|}{ Custos } & \multicolumn{2}{|c|}{ Moda } \\
\hline \multicolumn{2}{|c|}{ Quadro 1 - Relação das disciplinas de custos nos diferentes Cursos da IES pesquisada } \\
\hline
\end{tabular}


Os sujeitos da pesquisa foram os alunos que cursam a disciplina de custos, perfazendo o total de 289 alunos. Destes, 214 participaram da pesquisa por estar presente no momento da aplicação do instrumento da pesquisa, ocorrida no mês de outubro de 2009 , totalizando uma amostra de $74 \%$.

O instrumento utilizado para coleta de dados foi um questionário com base no modelo Kano adaptado de Walter, Tontini e Domingues (2005). A adaptação do questionário ocorreu na identificação e descrição dos atributos. Na pesquisa Walter, Tontini e Domingues (2005) o objetivo foi a identificação de atributos de qualidade relacionados a um curso de graduação de uma IES, enquanto nesta pesquisa o objetivo está na identificação de atributos de qualidade para a disciplina de custos.

Categorias como a capacitação dos professores tiveram atributos utilizados sem nenhuma adaptação nesta pesquisa, como atualização e titulação do professor. Porém, outras categorias e atributos necessitaram de adaptações direcionadas à disciplina de custos. Essas adaptações foram efetuadas com o auxílio da técnica de focus group.

Em relação à entrevista com grupos de foco, Mattar (1996, p. 64) a define como um método pouco estruturado, "conduzido por um moderador experiente, simultaneamente, com um pequeno número de participantes para obter dados sobre determinado assunto focalizado." Com o uso de um roteiro reuniu-se um grupo de 20 alunos inseridos na população pesquisada como forma de identificar atributos não contemplados no instrumento utilizado por Walter, Tontini e Domingues (2005), uma vez que este foi elaborado com foco de avaliação de um curso de uma IES e não específico a uma disciplina.

Da aplicação do focus group emergiram 3 atributos não contemplados no instrumento adaptado de Walter, Tontini e Domingues (2005), sendo: aplicação de diversos exercícios, aplicação de exercícios que envolvam indústria, comércio e serviços e a utilização de laboratório para resolução de exercícios em planilhas eletrônicas. Assim, o instrumento contemplou a possibilidade de análise de 23 atributos, agrupados em cinco categorias: capacitação dos professores, métodos de ensino, atitude, conteúdo e infra-estrutura, conforme mostra o Quadro 2.

\begin{tabular}{|c|l|c|}
\hline CATEGORIAS & \multicolumn{1}{|c|}{ ATRIBUTOS } & \multicolumn{1}{c|}{ FONTE } \\
\hline \multirow{4}{*}{$\begin{array}{c}\text { Capacitação dos } \\
\text { professores }\end{array}$} & Atualização do professor & Walter, Tontini e Domingues \\
\cline { 2 - 3 } & Titulação do professor & Walter, Tontini e Domingues \\
\cline { 2 - 3 } & Experiência prática sobre o conteúdo ministrado & Walter, Tontini e Domingues \\
\hline \multirow{4}{*}{$\begin{array}{c}\text { Métodos de } \\
\text { ensino }\end{array}$} & Relação entre teoria e prática na disciplina & Walter, Tontini e Domingues \\
\cline { 2 - 3 } & Aplicação de diversos exercícios & \multicolumn{1}{|c|}{ Focus Group } \\
\cline { 2 - 3 } & $\begin{array}{l}\text { Aplicação de exercícios que envolvam indústria, } \\
\text { comércio e serviços }\end{array}$ & $\begin{array}{l}\text { Utilização de laboratório para resolução de exercícios } \\
\text { em planilhas eletrônicas }\end{array}$ \\
\cline { 2 - 3 } & $\begin{array}{l}\text { A quantidade de materiais disponíveis no ambiente } \\
\text { virtual de aprendizagem }\end{array}$ & Walter, Tontini e Domingues \\
\cline { 2 - 3 } & Os critérios de avaliação usados na disciplina & Walter, Tontini e Domingues \\
\cline { 2 - 3 } & Nível de exigência do professor & Walter, Tontini e Domingues \\
\hline
\end{tabular}


Continuação

\begin{tabular}{|c|c|c|}
\hline CATEGORIAS & ATRIBUTOS & FONTE \\
\hline \multirow{4}{*}{ Atitude } & $\begin{array}{l}\text { Relacionamento do professor com os alunos em sala de } \\
\text { aula }\end{array}$ & Walter, Tontini e Domingues \\
\hline & Atendimento do professor em horários extraclasse & Walter, Tontini e Domingues \\
\hline & Disposição do professor para preparação das aulas & Walter, Tontini e Domingues \\
\hline & Disposição do professor para condução das aulas & Walter, Tontini e Domingues \\
\hline \multirow{6}{*}{ Conteúdo } & Contribuição da disciplina para o desempenho profissional & Walter, Tontini e Domingues \\
\hline & Pertinência dos assuntos para a disciplina & Walter, Tontini e Domingues \\
\hline & $\begin{array}{l}\text { Oferta de atiyidades extra-curriculares (Ex.: visitas à } \\
\text { empresa, estudo de campo) }\end{array}$ & Walter, Tontini e Domingues \\
\hline & Atualização do conteúdo & Walter, Tontini e Domingues \\
\hline & $\begin{array}{l}\text { Apresentação de inter-relação com as demais disciplinas } \\
\text { db curso }\end{array}$ & Walter, Tontini e Domingues \\
\hline & A carga horária da disciplina & Walter, Tontini e Domingues \\
\hline \multirow{3}{*}{ Infraestrutura } & Quantidade de títulos da disciplina de custos na biblioteca & Walter, Tontini e Domingues \\
\hline & $\begin{array}{l}\begin{array}{l}\text { Contribuiccão da modernidade dos laboratórios de } \\
\text { informatica para a disciplina }\end{array} \\
\end{array}$ & Walter, Tontini e Domingues \\
\hline & $\begin{array}{l}\text { A infraestrutura da sala de aula (tamanho, conforto, } \\
\text { ventiladores el ou ar condicionado, equipamentos) }\end{array}$ & Walter, Tontini e Domingues \\
\hline
\end{tabular}

Quadro 2 - Categorias de atributos utilizados na pesquisa

Fonte: Adaptado de Walter, Tontini e Domingues (2005) e complementadas pelo Focus Group.

O questionário foi composto por quatro etapas. A primeira identificou atributos segundo o modelo Kano. Nesse sentido, sempre havia duas perguntas apresentadas aos sujeitos da pesquisa, descrevendo respectivamente uma situação de aumento e outra de diminuição no desempenho de cada atributo. Para cada pergunta, o respondente indicou sua expectativa de satisfação ou insatisfação em relação às situações mencionadas, numa escala variando de extremamente insatisfeito a extremamente satisfeito, demonstrado no Quadro 3.

\begin{tabular}{|c|c|c|c|c|c|c|c|c|c|c|c|}
\hline Descreva como você se SENTIRIA: & \multicolumn{6}{|c|}{ Muito Insatisfeito - Neutro -Muito Satisfeito } \\
\cline { 2 - 10 } ATRIBUTOS & -5 & -4 & -3 & -2 & -1 & 0 & $\mathbf{1}$ & $\mathbf{2}$ & $\mathbf{3}$ & $\mathbf{4}$ & $\mathbf{5}$ \\
\hline Se o professor da disciplina se mostrar mais atualizado & & & & & & & & & & & \\
\hline Se o professor da disciplina se mostrar menos atualizado & & & & & & & & & & & \\
\hline
\end{tabular}

Quadro 3 - Questões para identificação dos atributos pelo Modelo Kano de Qualidade Atrativa e Obrigatória

Na segunda parte mediu-se a satisfação geral atual com a disciplina de custos e o grau de satisfação existente em relação ao nível de desempenho atual encontrado nos atributos. Utilizou-se também uma escala que variou de -5 (extremamente insatisfeito) a +5 (extremamente satisfeito). Já na terceira etapa verificou-se o grau de importância para cada um dos atributos do curso, na qual os alunos atribuíram notas numa escala de importância de 1 a 5 . Na última etapa, pesquisaram-se dados pessoais e de frequência de utilização dos atributos, por meio de perguntas fechadas. 
O questionário foi desenvolvido eletronicamente, utilizando-se da ferramenta do Google Docs. Para a aplicação do questionário utilizou-se do laboratório de informática da IES. Cada etapa do questionário foi explicada aos alunos previamente antes da liberação dos questionários aos mesmos. À medida que cada etapa foi respondida, as tabulações em planilha eletrônica ocorreram de forma simultânea para realização da análise posterior.

Os resultados obtidos por meio do questionário foram plotados em um gráfico de dispersão com auxílio de uma planilha eletrônica. Para divisão dos quadrantes da matriz de importância e desempenho, optou-se por estabelecer a linha divisória de importância em 3 (importante na escala do questionário) e a linha da satisfação atual pela média geral da satisfação atual de todos os atributos pesquisados, que foi de 2. Já para o gráfico de dispersão do índice de satisfação do cliente conforme o modelo Kano, estabeleceu-se a divisão dos quadrantes em 0,4 para satisfação e - 0,4 para insatisfação.

\section{ANÁLISE E INTERPRETAÇÃO DOS DADOS}

Na Figura 1, apresenta-se o gráfico de dispersão para análise da Matriz de Importância e Desempenho para os atributos pesquisados.

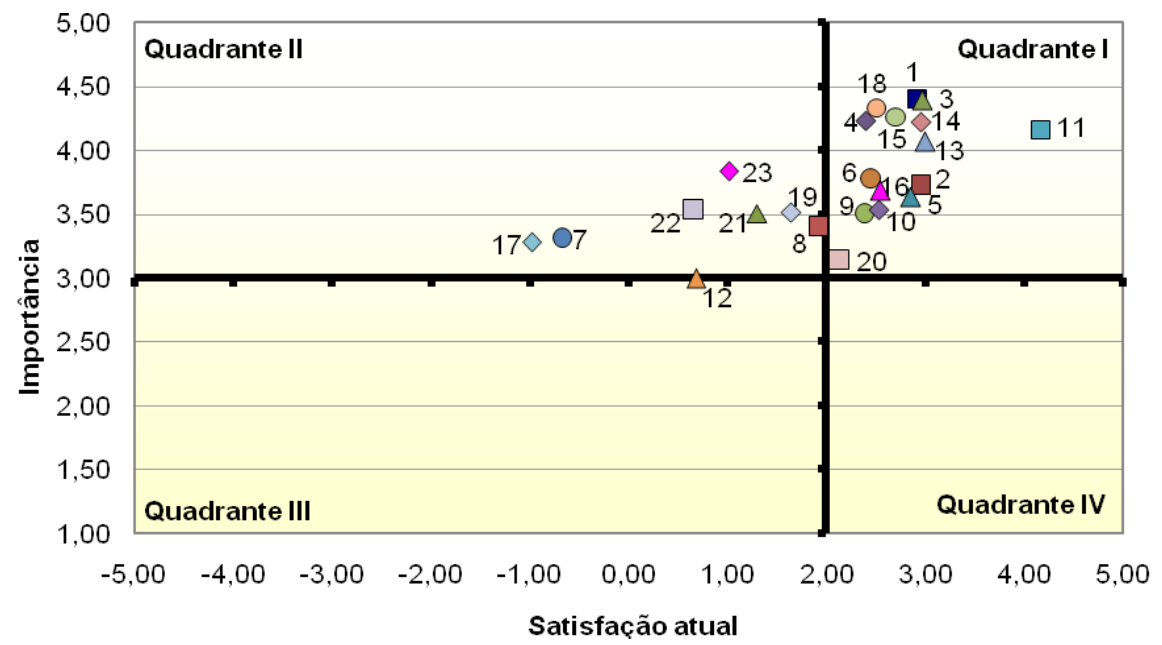

Legenda:

\begin{tabular}{|l|l|l|l|}
\hline 1. & Atualização do professor & 13. & Disposição do professor para preparar as aulas \\
\hline 2. & Titulação do professor & 14. & Disposição do professor para conduzir as aulas \\
\hline 3. & Experiência prática do professor no assunto ministrado & 15. & Contribuição da disciplina para o desempenho profissional \\
\hline 4. & Relação entre teoria e prática & 16. & Pertinência dos assuntos para a disciplina \\
\hline 5. & Quantidade de exercícios & 17. & Oferta de atividades extracurriculares \\
\hline 6. & Aplicação de exercícios que envolvam indústria, comércio e serviços & 18. & Atualização do conteúdo \\
\hline 7. & Utilização de laboratório para resolução de exercícios & 19. & Apresentação de interrelação com as demais disciplinas do curso \\
\hline 8. & Quantidade de materiais disponíveis no ambiente virtual de aprendizagem & 20. & Carga horária da disciplina \\
\hline 9. & Critérios de avaliação usados na disciplina & 21. & Quantidade de títulos da disciplina de custos na biblioteca \\
\hline 10. & Nível de exigência do professor & 22. & Modernidade dos laboratórios de informática \\
\hline 11. & Relacionamento do professor com os alunos em sala de aula & 23. & Infraestrutura da sala de aula \\
\hline 12. & Atendimento do professor em horários extraclasse & & \\
\hline
\end{tabular}


No Quadrante I da Figura 1, situam-se os atributos de alta importância e de alta satisfação, ou seja, os pontos fortes da disciplina de contabilidade de custos, sendo eles:

- (11) relacionamento do professor com os alunos em sala de aula, (1) atualização do professor,

- (3) experiência prática do professor no assunto ministrado, (14) disposição do professor para conduzir as aulas,

- (13) disposição do professor para preparar as aulas, (2) titulação do professor, (5) quantidade de exercícios,

- (15) contribuição da disciplina para o desempenho profissional, (16) pertinência dos assuntos para a disciplina,

- (10) nível de exigência do professor, (4) relação entre teoria e prática,

- (6) aplicação de exercícios que envolvam indústria, comércio e serviços, (9) critérios de avaliação usados na disciplina e

- (20) carga horária da disciplina. Destes atributos, (11) relacionamento do professor com os alunos em sala de aula se destaca como o de maios satisfação e

- (20) carga horária da disciplina situa-se quase na média de satisfação.

No Quadrante II, encontram-se os atributos considerados de alta importância e que estão com o nível de satisfação abaixo da média. Estes são os atributos que, segundo a matriz de importância e desempenho, merecem melhorias:

- (17) oferta de atividades extracurriculares, (7) utilização de laboratório para resolução de exercícios,

- (22) modernidade dos laboratórios de informática para a disciplina, (23) infraestrutura da sala de aula,

- (21) quantidade de títulos da disciplina de custos na biblioteca, (19) apresentação de interrelação com as demais disciplinas do curso e

- (8) quantidade de materiais disponíveis no ambientevirtual de aprendizagem. 0 atributo (17) oferta de atividades extracurriculares é o que se apresenta com a satisfação atual mais baixa e o

- (23) infraestrutura da sala de aula com a importância mais alta. Além disso, o atributo (19) apresentação de interrelação com as demais disciplinas do curso apresenta-se quase na média de satisfação.

Tem-se ainda o atributo (12) atendimento do professor em horários extraclasse, que se apresenta na média de importância e com a satisfação atual abaixo da média. O fato de este ser o único atributo a não ter importância superior a três pode estar 
relacionado a muito alunos não terem o hábito de procurar o professor fora do horário de aula.

Na Figura 2, destaca-se o gráfico de dispersão para análise do índice de satisfação do cliente conforme o modelo Kano de Qualidade Atrativa e Obrigatória.

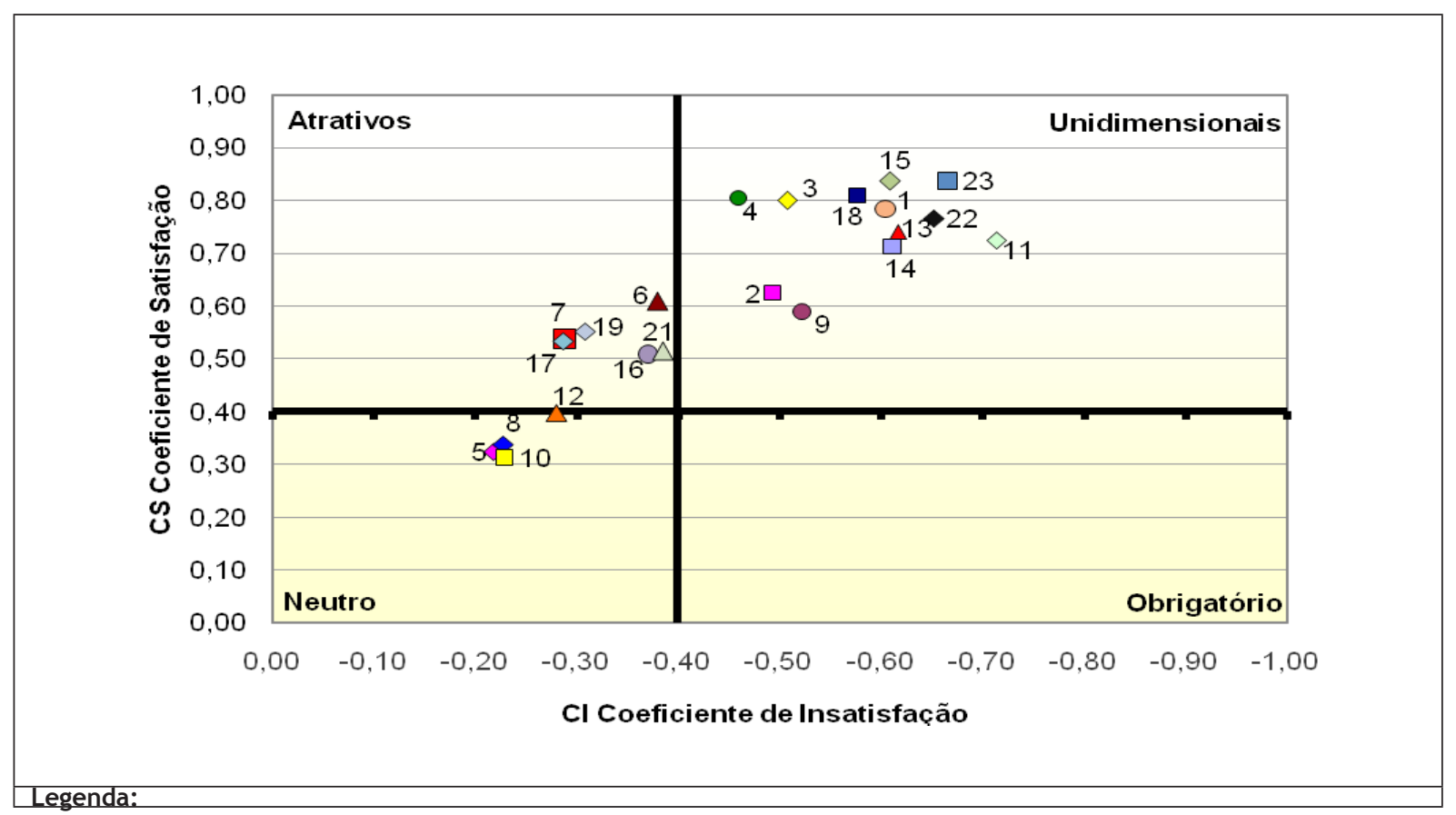

\begin{tabular}{|c|l|c|l|}
\hline 1 & Atualização do professor & 13 & Disposição do professor para preparar as aulas \\
\hline 2 & Titulação do professor & 14 & Disposição do professor para conduzir as aulas \\
\hline 3 & $\begin{array}{l}\text { Experiência prática do professor no assunto } \\
\text { ministrado }\end{array}$ & 15 & $\begin{array}{l}\text { Contribuição da disciplina para o desempenho } \\
\text { profissional }\end{array}$ \\
\hline 4 & Relação entre teoria e prática & 16 & Pertinência dos assuntos para a disciplina \\
\hline 5 & Quantidade de exercícios & 17 & Oferta de atividades extracurriculares \\
\hline 6 & $\begin{array}{l}\text { Aplicação de exercícios que envolvam indústria, } \\
\text { comércio e servicos }\end{array}$ & 18 & Atualização do conteúdo \\
\hline 7 & $\begin{array}{l}\text { Utilização de laboratório para resolução de } \\
\text { exerćios }\end{array}$ & 19 & $\begin{array}{l}\text { Apresentação de interrelação com as demais } \\
\text { disciplinas do curso }\end{array}$ \\
\hline 8 & $\begin{array}{l}\text { Quantidade de materiais disponiveis no ambiente } \\
\text { virtual de aprendizagem }\end{array}$ & 20 & Carga horária da disciplina \\
\hline 9 & Critérios de avaliação usados na disciplina & 21 & $\begin{array}{l}\text { Quantidade de titulos da disciplina de custos na } \\
\text { biblioteca }\end{array}$ \\
\hline 10 & Nível de exigência do professor & 22 & Modernidade dos laboratórios de informática \\
\hline 11 & $\begin{array}{l}\text { Relacionamento do professor com os alunos em } \\
\text { sala de aula }\end{array}$ & 23 & Infraestrutura da sala de aula \\
\hline 12 & $\begin{array}{l}\text { Atendimento do professor em horários } \\
\text { extraclasse }\end{array}$ & \multicolumn{2}{|l}{} \\
\hline
\end{tabular}

Figura 2 - Gráfico do índice de satisfação do cliente

Por meio da Figura 2, observa-se que três atributos podem ser considerados como neutros, ou seja, para os alunos seu desempenho é indiferente: (5) quantidade de exercícios, (8) quantidade de materiais disponíveis no ambiente virtual de aprendizagem e (10) nível de exigência do professor. Uma possível explicação para este resultado pode ser os alunos utilizarem pouco o ambiente de aprendizagem e não se importarem com uma possível alteração na quantidade de exercícios ou no nível de exigência, desde que percebam que isso está alinhado ao conteúdo e à aprendizagem proporcionada.

Tem-se ainda o atributo (12) atendimento do professor em horários extraclasse, que se apresentou na linha divisória entre neutro e atrativo. Este resultado corrobora 
a hipótese levantada sobre a parte dos alunos não procurarem o professor fora do horário de aula para atendimento. Assim, para os alunos que procuram este serviço, ele pode ser um atrativo em virtude de seu diferencial, já para os alunos que não utilizam o serviço, ele se torna indiferente.

Entre os atributos classificados como unidimensionais, sendo a satisfação proporcional ao nível de desempenho tem-se: (4) relação entre teoria e prática, (2) titulação do professor, (3) experiência prática do professor no assunto ministrado, (9) critérios de avaliação usados na disciplina, (18) atualização do conteúdo, (15) contribuição da disciplina para o desempenho profissional, (1) atualização do professor, (13) disposição do professor para preparar as aulas, (14) disposição do professor para conduzir as aulas, (23) infraestrutura da sala de aula, (22) modernidade dos laboratórios de informática e (11) relacionamento do professor com os alunos em sala de aula.

Têm-se também os atributos considerados como atrativos, os quais proporcionam uma satisfação mais que proporcional quando estão com desempenho acima da média, mas não causam insatisfação quando estão com desempenho abaixo da média, são eles: (17) oferta de atividades extracurriculares, (7) utilização de laboratório para resolução de exercícios, (19) apresentação de interrelação com as demais disciplinas do curso, (6) aplicação de exercícios que envolvam indústria, comércio e serviços, (16) pertinência dos assuntos para a disciplina e (21) quantidade de títulos da disciplina de custos na biblioteca.

Por meio da análise conjunta dos resultados obtidos para a matriz de importância e desempenho e para o modelo Kano, pode-se definir com maior segurança as prioridades de melhoria para a disciplina. Dos atributos identificados como importantes e abaixo da média de satisfação na matriz de importância e desempenho, que seriam prioridades de melhoria se considerado este método isoladamente, (8) quantidade de materiais disponíveis no ambiente virtual de aprendizagem foi identificado como neutro, ou seja, não causa satisfação nem insatisfação se tiver seu desempenho alterado.

Nesse sentido, as prioridades principais de melhoria passam a ser os atributos unidimensionais com alta importância e abaixo da média de satisfação, visto que atualmente estão causando insatisfação, sendo eles: (22) modernidade dos laboratórios de informática para a disciplina e (23) infraestrutura da sala de aula. Nota-se que estes atributos envolvem investimentos financeiros e estão além da alçada do professor da disciplina, dependendo da universidade e de suas disponibilidades de recursos.

Em um segundo momento, pode-se investir nos atributos atrativos importantes e abaixo da média de satisfação, visto que sua melhoria trará satisfação mais que proporcional, apesar do baixo desempenho não causar insatisfação. Estes foram identificados como: (17) oferta de atividades extracurriculares, (7) utilização de laboratório para resolução de exercícios, (21) quantidade de títulos da disciplina de custos na biblioteca e (19) apresentação de interrelação com as demais disciplinas do curso. Já no tocante a estes atributos, alguns são de cunho da instituição, como quantidade de títulos da disciplina de custos na biblioteca; outros do professor, como apresentação de interrelação com as demais disciplinas do curso; e ainda têm- 
se os que dependem tanto da iniciativa do professor quanto da disponibilidade de infraestrutura da universidade ou de autorização desta, como utilização de laboratório para resolução de exercícios e oferta de atividades extracurriculares.

\section{CONCLUSÕES}

A pesquisa teve por objetivo aplicar a técnica do modelo Kano de Qualidade Atrativa e Obrigatória em conjunto com a Matriz de Importância e Desempenho para identificar oportunidades de melhoria na disciplina de custos de diferentes cursos de graduação.

Ela foi realizada por meio de um estudo descritivo, de levantamento com uma abordagem qualitativa e quantitativa. Utilizou-se um questionário que foi aplicado em 214 alunos de 9 turmas em 5 cursos: administração, ciências contábeis, ciências econômicas, engenharia de produção e moda. O questionário contemplou a possibilidade de análise de 23 atributos, agrupados em cinco categorias, sendo a capacitação dos professores, os métodos de ensino, a atitude, o conteúdo e a infraestrutura.

$\mathrm{Na}$ identificação de oportunidades de melhoria na disciplina de custos verificaram-se atributos constantes em três quadrantes: neutros, atrativos e unidimensionais.

No quadrante neutro, no qual o desempenho é indiferente para os alunos, constataram-se três atributos na amostra pesquisada, sendo a quantidade de exercícios, a quantidade de materiais disponíveis no ambiente virtual de aprendizagem e o nível de exigência do professor.

No quadrante dos atributos considerados como atrativos, os quais proporcionam uma satisfação mais que proporcional quando estão com desempenho acima da média, mas não causam insatisfação quando estão com desempenho abaixo da média, identificaram-se seis atributos: oferta de atividades extracurriculares, utilização de laboratório para resolução de exercícios, apresentação de interrelação com as demais disciplinas do curso, aplicação de exercícios que envolvam indústria, comércio e serviços, pertinência dos assuntos para a disciplina e quantidade de títulos da disciplina de custos na biblioteca.

O quadrante unidimensional, no qual a satisfação foi proporcional ao nível de desempenho, foi o quadrante que mais apresentou atributos, num total de 12 , sendo: relação entre teoria e prática, titulação do professor, experiência prática do professor no assunto ministrado, critérios de avaliação usados na disciplina, atualização do conteúdo, contribuição da disciplina para o desempenho profissional, atualização do professor, disposição do professor para preparar as aulas, disposição do professor para conduzir as aulas, infraestrutura da sala de aula, modernidade dos laboratórios de informática e relacionamento do professor com os alunos em sala de aula.

Ao efetuar a análise conjunta dos resultados obtidos para a matriz de importância e desempenho com o modelo Kano, identificou-se com maior segurança prioridades 
de melhoria para a disciplina de custos. Dos atributos identificados como importantes e abaixo da média de satisfação na matriz de importância e desempenho, que são prioridades de melhoria se considerado este método isoladamente, a quantidade de materiais disponíveis no ambiente virtual de aprendizagem foi identificado como neutro, ou seja, não causa satisfação nem insatisfação se tiver seu desempenho alterado.

Identificaram-se os atributos que atualmente causam insatisfação, ou seja, com alta importância e abaixo da média de satisfação. Estes atributos são identificados no quadrante dos atributos unidimensionais, que são: a modernidade dos laboratórios de informática para a disciplina e a infraestrutura da sala de aula. Observa-se que a melhoria desses atributos envolverá investimentos financeiros da universidade para adequação a satisfação dos alunos.

Identificou-se também, por meio dos atributos atrativos importantes e abaixo da média de satisfação, possibilidades de ações que poderão trazer satisfação mais que proporcional, apesar do baixo desempenho não causar insatisfação. Os atributos identificados foram a oferta de atividades extracurriculares, a utilização de laboratório para resolução de exercícios, a quantidade de títulos da disciplina de custos na biblioteca e a apresentação de interrelação com as demais disciplinas do curso.

Verifica-se, assim, que a aplicação do Modelo Kano de qualidade aplicado em conjunto com a matriz de importância e desempenho possibilitou uma visão sobre quais atributos pertinentes a disciplina de custos devem ser melhorados para proporcionar satisfação aos alunos, dentro de uma perspectiva de atributos considerados obrigatórios, unidimensionais e atrativos.

Como se trata de um grupo específico que cursou a disciplina é oportuno continuar a pesquisa para novos grupos de alunos que farão as disciplinas de custos e, com base numa séria histórica, observar aderência ou novos achados a presente pesquisa. Também é possível a implementação de alguns atributos julgados necessários de melhoria nessa pesquisa e verificar se o atendimento desses atributos será perceptível numa pesquisa futura. 


\section{REFERÊNCIAS}

BERGER, Charles et al. Kano's Methods for Understanding Customer-Defined Quality. Journal of the Japanese Society for Quality Control, v. 23, 2, p. 3-35, 1993.

BEUREN, Ilse Maria e KLANN, Roberto Carlos. Análise dos reflexos das divergências entre IFRS e US GAAP na evidenciação contábil de empresas inglesas listadas na LSE. In. Congresso USP de Controladoria e Contabilidade, 8, 2008, são Paulo. Anais... São Paulo, 2008. 1 CD-ROM.

BOUDON, Raymond. Os métodos em sociologia. São Paulo: Ática, 1989.

DESCHAMPS, Marcelo. Avaliação de qualidade no ensino superior: aplicação dos modelos HEdPERF e SERVPERF na Faculdade de Tecnologia do Senai/SC-Blumenau. 2007. 194f. Mestrado (Programa de Pós-Graduação em Administração) - Centro de Ciências Sociais Aplicadas, Universidade Regional de Blumenau, Blumenau, 2007.

FIEBIG, Evelyn Quadros. Avaliação de cursos de graduação em administração: proposta de uma metodologia a partir de um estudo de caso em Florianópolis focalizando o consumidor no papel de acadêmico. 2006. 107f. Dissertação (Mestrado em Administração) - Cursos de Pós-Graduação em Administração, Universidade Federal de Santa Catarina, Florianópolis, 2006.

FIGUEREDO, Marcelo Salmeron. Percepções sobre os atributos de qualidade da Associação Educacional do Vale do Itajaí Mirim a partir da integração do modelo Servqual e Kano. 2005. 165 f. Dissertação (Mestrado em Administração) - Centro de Ciências Sociais Aplicadas, Universidade Regional de Blumenau, Blumenau, 2005.

GARVER, Michael S. Best practices in identifying customer-driven improvement opportunities. Industrial Marketing Management, v. 32, p. 455-466, 2003.

GIL, Antonio Carlos. Como elaborar projetos de pesquisa. 4. ed. São Paulo: Atlas, 2002.

KANO, N. et al. Attractive quality and must-be quality. The Journal of Japanese Society for Quality Control, v. 14, p. 39-48, abr. 1984.

MAINARDES, Emerson Wagner. Atração e retenção de alunos em cursos de graduação em administração das instituições particulares de ensino superior de Joinville/ SC. 2007. 331f. Dissertação (Mestrado em Administração) - Centro de Ciências Sociais Aplicadas, Universidade Regional de Blumenau, Blumenau, 2007.

MARTILLA, J. A.; JAMES, J. C. Importance-performance analysis. Journal of Marketing, New York, v. 41, 1, p. 77-79, jan. 1977.

MARZO-NAVARRO, Mercedes; PEDRAJA-IGLESIAS, Marta; RIVERA-TORRES, M. Pilar. Measuring customer satisfaction in summer courses. Quality Assurance in Education, Bradford, v. 13, 1, p. 64, 2005. 
MATTAR, Fauze N. Pesquisa de marketing. São Paulo: Atlas, 1996. Edição compacta.

MATZLER, Kurt. et al. How to delight your customers. Journal of Product \& Brand Management, v. 5, 2, p. 6-18, 1996.

MATZLER, Kurt. et al. The asymmetric relationship between attribute-level performance and overall customer satisfaction: a reconsideration of the importanceperformance analysis. Industrial marketing Management. v. 33, p. 271-277, 2004. DOI:10.1016/S0019-8501(03)00055-5.

MONDINI, Vanessa Edy Dagnoni. Estudo da satisfação dos alunos dos programas de pós-graduação em Administração em Santa Catarina e no Paraná. 2006. 141f. Dissertação (Mestrado em Administração) - Centro de Ciências Sociais Aplicadas, Universidade Regional de Blumenau, Blumenau, 2006.

MONDINI, Vanessa Edy Dagnoni; SILVEIRA, Amélia; TONTINI, Gérson. Atributos de satisfação no contexto da pós-graduação em Administração: a ótica dos discentes. In: SIMPÓSIO DE GESTÃO E ESTRATÉGIÁ EM NEGÓCIOS, 5, 2007, Seropédica. Anais... Seropédica, UFRuralRJ, 2007.

MORALES, Miguel; MONCLOA, Luis Felipe Calderón. Testing the construct validity of an instrument to measure executive education service quality: a follow-up study in Latin America. In: THE BUSINESS ASSOCIATION OF LATIN AMERICAN STUDIES, 2005, Madrid. Proceedings...Madri: BALAS, 2005.

PARISOTTO, lara Regina dos Santos et al. Identificando oportunidades de melhoria no curso de ciências contábeis com a utilização conjunta do modelo Kano de qualidade e da matriz de importância x desempenho. In. Congresso ANPCONT, 2, 2008, Salvador. Anais... Salvador, 2008. 1 CD-ROM.

RICHARDSON, Roberto Jarry. Pesquisa social: métodos e técnicas. São Paulo: Atlas, 1989.

SILVEIRA, Amelia; APPIO, Jucelia; DOMINGUES, Maria José Carvalho de Souza. Atributos de qualidade da disciplina de custos aplicados à administração. In. CONGRESSO BRASILEIRO DE CUSTOS, 15, 2008. Curitiba. Anais... Curitiba: 2008. 1 CD-ROM.

TONTINI, Gerson. Como identificar atributos atrativos e obrigatórios para o consumidor. Revista de Negócios, Blumenau, v. 8, 1, p. 19-28, $2003 a$.

TONTINI, Gerson. Determining the Degree of satisfaction of customer requirements: a modified Kano method, California Journal of Operations Management, Turlock California - EUA, v. 1, 1, p. 95 - 103, 2003b.

TONTINI, Gerson, et al. Análise de oportunidades de melhoria em laboratórios fotográficos através da integração da matriz de importância x desempenho com o modelo Kano de qualidade. Revista de Negócios, Blumenau, v. 9, 3, p. 179-188, jul./ set. 2004.

TONTINI, Gérson; SILVEIRA, Amélia . Identification of critical attributes of success in products and services: an alternative to importance - performance analysis. In: BALAS 
ANNUAL CONFERENCE, 2005, Madrid. Proceedings... Madrid: Instituto de Empresa, 2005. p. 1-15.

WALTER, Silvana Anita; TONTINI, Gérson; DOMINGUES, Maria José Carvalho de Souza. Identificando oportunidades de melhoria em um curso superior através da análise da satisfação dos alunos. In: ENCONTRO NACIONAL DE PROGRAMAS DE PÓS-GRADUAÇÃO EM ADMINISTRAÇÃO, 29, 2005, Brasília. Anais... Porto Alegre: Pallotti, 2005. 1 CDROM.

WALTER, Silvana Anita. Antecedentes da satisfação e da lealdade de alunos de uma instituição de ensino superior. 2006. 167 f. Dissertação (Mestrado em Administração) - Centro de Ciências Sociais Aplicadas, Universidade Regional de Blumenau, Blumenau, 2006.

ZANI, Adriana Valongo. Incidentes críticos do processo ensino-aprendizagem do curso de graduação em enfermagem segundo a percepção de alunos e professores. 2005. 191f. Dissertação (Mestrado em Enfermagem) - Programa de Pós-Graduação em Enfermagem de Ribeirão Preto, Universidade de São Paulo, Ribeirão Preto, 2005.

ZWIEREWICZ, Márcia. Atributos de qualidade em cursos de pós-graduação em administração do estado de Santa Catarina: estudo com a técnica do incidente crítico. 2008. 188f. Dissertação (Mestrado em Administração) - Centro de Ciências Sociais Aplicadas, Universidade Regional de Blumenau, Blumenau, 2008. 


\section{ENDEREÇO DOS AUTORES:Paulo Roberto da Cunha}

Universidade do Estado de Santa Catarina

Centro de Educação Superior do Alto Vale do Itajaí - CEAVI.

Rua Dr. Getúlio Vargas, 2822 - Bela Vista

Ibirama, SC - Brasi

89140-000

\section{Roberto Carlos Klann}

Fundação Educacional de Brusque

Centro Universitário de Brusque Unifebe, Curso de Ciências Contábeis.

Rua Dorval Luz, 647 - Santa Terezinha

Brusque, SC - Brasil

$88352-400$

Júlio Orestes da Silva

Fundação Universidade Regional de Blumenau.

Rua Antônio da Veiga, 140 - Victor Konder

Blumenau, SC - Brasil

89012-900

Jorge Eduardo Scarpin

Fundação Universidade Regional de Blumenau

Programa de Pós Graduação em Ciências Contábeis.

Rua Antônio da Veiga, 140 - Victor Konder

Blumenau, SC - Brasil

89012-900 